\title{
Prevalência e fatores associados à ideação suicida na adolescência: revisão de literatura
}

\author{
Lenice Carrilho de Oliveira Moreira \\ Universidade Federal de Mato Grosso do Sul - Campo Grande - MS \\ Paulo Roberto Haidamus de Oliveira Bastos \\ Universidade Federal de Mato Grosso do Sul - Campo Grande - MS
}

\begin{abstract}
Resumo
Para a elaboração deste artigo foi realizada uma revisão da literatura sobre a prevalência e os principais fatores associados à ideação suicida em adolescentes da população não clínica. A revisão foi baseada nos estudos de corte transversal encontrados em bases de dados eletrônicas (Medline, SciELO e Lilacs), referentes ao período de 2002 a 2013. As palavras-chave utilizadas foram: "ideação suicida", "ideação suicida e adolescentes", "ideação suicida e estudantes", "comportamento suicida e adolescentes". A prevalência de ideação suicida é alta e está significativamente relacionada a fatores como: depressão, uso de álcool e drogas, violência física, problemas de relacionamento com os pais, tristeza e solidão. A ideação suicida associada à depressão em adolescentes é prevenível desde que o adolescente seja devidamente tratado. Há necessidade de investimentos em pesquisas no Brasil, dado o crescimento das taxas de suicídio entre adolescentes, sobretudo do sexo masculino.
\end{abstract}

Palavras-chave: Comportamento autodestrutivo; suicídio; adolescentes.

\section{Prevalence and risk factors associated with suicidal ideation in adolescents: literature review}

\begin{abstract}
To prepare this article a review in the literature was made focusing on the prevalence and risk factors associated with suicidal ideation in adolescents from non-clinical population. The revision was based on cross-sectional studies found in electronic databases (Medline, SciELO and Lilacs) for the period 2002 to 2013. The key words used were "suicidal ideation", "suicidal ideation and adolescents" "suicidal ideation and students", "suicide and adolescent behavior." The prevalence of suicidal ideation is high and is significantly related to factors such as: depression, alcohol and drug use, physical violence, relationship problems with parents, sadness and loneliness. Suicidal ideation associated with depression in adolescents is preventable since the teenager is properly treated. There is need for investment in research in Brazil, given the increase in suicide rates among adolescents, especially males.
\end{abstract}

Keywords: Self destructive behavior; Suicide; teens.

\section{Prevalencia y factores asociados a la ideación suicida en la adolescencia: revisión de literatura}

\section{Resumen}

Para la elaboración de este artículo se realizó una revisión da literatura sobre la prevalencia y los principales factores asociados a la ideación suicida em adolescentes de la populación no clínica. Se basó la revisión en los estudios de corte transversal encontrados en bases de datos electrónicas (Medline, SciELO y Lilacs), referentes al período de 2002 a 2013 . Se utilizaron las palabras clave: "ideación suicida", "ideación suicida y adolescentes", "ideación suicida y estudiantes", "comportamiento suicida y adolescentes". La prevalencia de ideación suicida es alta y está significativamente relacionada a factores como: depresión, uso de alcohol y drogas, violencia física, problemas de relacionamiento com los padres, tristeza y soledad. La ideación suicida asociada a la depresión en adolescentes es prevenible desde que el adolescente sea debidamente tratado. Hay la necesidad de inversiones em investigaciones en Brasil, dado al crecimiento de las tasas de suicidio entre adolescentes, sobre todo del sexo masculino.

Palabras clave: Comportamiento autodestructivo; suicidio; adolescentes 


\section{Introdução}

A morte é temida pela maioria das pessoas; entretanto, pode ser considerada como um alívio para aqueles que não encontram alternativas para seus problemas, e buscam, por meio de comportamentos autodestrutivos, acabar com a própria vida. Por ser um período de desenvolvimento marcado por diversas modificações biológicas, psicológicas e sociais que, geralmente, são acompanhadas de conflitos e angústias, tem-se observado, nas últimas décadas, um crescimento no comportamento suicida entre jovens (Araújo, Vieira, \& Coutinho, 2010). Segundo Borges e Werlang (2006a), a idade de 15 anos é considerada crítica para manifestações de comportamento suicida na adolescência.

As estatísticas sobre o suicídio são falhas e subestimadas, principalmente com relação aos adolescentes, visto que seus atos autodestrutivos são, muitas vezes, negados e escondidos pela família (Borges, \& Werlang, 2006a). Classificado como "causas externas", o suicídio entre adolescentes jovens é pouco investigado, vez que sua etiologia é complexa e envolve, além de fatores biológicos e psicológicos, o contexto socioeconômico (Castro, Cunha, \& Souza, 2011).

Uma vez que a maioria das pesquisas referentes à ideação suicida e tentativa de suicídio utiliza amostras clínicas, buscamos identificar, na literatura, pesquisas que apresentam a prevalência e fatores associados à ideação suicida na adolescência, com base na população geral, dando ênfase especial às pesquisas realizadas com adolescentes escolares.

\section{Metodologia}

Foi realizada uma revisão narrativa e compreensiva de estudos e pesquisas sobre a prevalência e fatores associados à ideação suicida em adolescentes, considerando-se, também, as tentativas e o suicídio consumado, vez que há uma estreita relação entre estes três momentos.

As referências apresentadas pela literatura sobre a ideação suicida em adolescentes foram coletadas a partir das bases de dados Medline, SciELO e Lilacs. A busca foi limitada aos estudos de corte transversal, publicados no período compreendido entre 2002 e 2013, nos idiomas português, inglês e espanhol. Foram analisados, ainda, estudos que investigaram o risco suicida e pensamentos de automutilação nos adolescentes, pois ambos, assim como a ideação suicida, envolvem atitudes, desejos e manifestações da intenção de querer morrer.

Os descritores utilizados na busca da revisão foram: "prevalência de ideação suicida", "ideação suicida e adolescentes", "ideação suicida e estudantes", "comportamento suicida e adolescentes", "comportamento suicida e estudantes", nos idiomas português e inglês. Como resultado dessa estratégia de busca encontrou-se um total de 3.250 artigos nas bases selecionadas.

Estabeleceu-se que, para serem selecionados, os artigos deveriam preencher os seguintes critérios: 1) identifi- car a prevalência de ideação/risco suicida em adolescentes escolares da população não clínica; 2) identificar os fatores associados à ideação suicida em adolescentes; e 3) ter um desenho transversal. Os resumos foram analisados e os artigos com texto completo que preencheram os critérios de inclusão foram recuperados.

Foram analisadas 33referências sobre o tema proposto, que preencheram todos os critérios estabelecidos. Outras onze referências, embora não estivessem relacionadas diretamente à prevalência ou fatores associados à ideação suicida na adolescência, foram incluídas nesta revisão, em função de trazerem informações importantes ao tema, tais como finalidades, conceitos, estatísticas de suicídio, normas e legislações específicas.

Os dados foram sistematizados em três categorias: 1) comportamento suicida; 2) dimensão do suicídio no Brasil; e 3) prevalência de ideação suicida em adolescentes.

\section{Comportamento suicida}

Todo e qualquer ato por meio do qual uma pessoa causa lesão a si própria, independente do grau de letalidade, é considerado comportamento suicida. O comportamento suicida classifica-se em três categorias distintas: ideação suicida, tentativa de suicídio e suicídio consumado. A ideação suicida fica em um dos extremos, o suicídio consumado no outro e a tentativa de suicídio entre eles (Werlang, Borges, \& Fensterseifer, 2005).

A palavra suicídio deriva etimologicamente do latim sui (si mesmo) e caedes (ação de matar) e significa uma morte intencional autoinfligida. Relatos apontam que o comportamento suicida existe desde os tempos mais antigos da humanidade, tendo mudado apenas a forma como esse ato é encarado (Correa, \& Barrero, 2006a).

De acordo com Lovisi, Santos, Legay, Abelha e Valencia (2009), os principais fatores associados ao suicídio são: tentativas anteriores de suicídio, doenças mentais (principalmente depressão e abuso/dependência de álcool e drogas), ausência de apoio social, histórico de suicídio na família, forte intenção suicida, eventos estressantes e características sociais e demográficas, tais como pobreza, desemprego e baixo nível educacional. Embora não exista uma definição única aceitável, o suicídio implica necessariamente um desejo consciente de morrer e a noção clara do que o ato executado pode resultar (Araújo, \& cols., 2010).

Dados da Organização Mundial da Saúde (OMS) demonstram que a frequência do suicídio está se deslocando dos idosos para os mais jovens. A maioria dos suicídios ocorre entre crianças maiores de 14 anos, principalmente no início da adolescência, entretanto, está ocorrendo, em alguns países, um aumento alarmante nos suicídios entre crianças menores de 15 anos, bem como na faixa etária dos 15 aos 19 anos (OMS, 2000).

A tentativa de suicídio é o ato sem resultado letal no qual o indivíduo deliberadamente causa danos a si mesmo, 
porém é considerado o maior predito clínico de futura tentativa de morte por suicídio (Correa, \& Barrero, 2006b).

Considerando que os registros oficiais sobre tentativas de suicídio são mais escassos e menos confiáveis do que os de suicídio, o número de tentativas de suicídio pode superar, em pelo menos dez vezes, o de suicídios (Brasil, 2006b).

Ideação suicida se refere aos pensamentos de autodestruição e ideias suicidas, englobando desejos, atitudes e planos que o indivíduo tem para dar fim à própria vida (Borges, \& Werlang, 2006b).Ter pensamentos suicidas uma vez ou outra não é anormal, vez que estes pensamentos fazem parte do processo de desenvolvimento normal da passagem da infância para a adolescência, à medida que se lida com problemas existenciais e se está tentando compreender a vida, a morte e o significado da existência (OMS, 2000).

A adolescência é uma fase em que ocorrem modificações psicológicas, físicas e sociais, sendo comum, nessa fase, acontecerem movimentos de dependência e independência extrema, caracterizando um período de contradições, conflitos e ambivalências. No entanto, muitos dos comportamentos atípicos manifestados pelos adolescentes podem ser apenas uma busca de sua identidade, sendo naturalmente superados.

Os pensamentos suicidas tornam-se anormais quando a realização destes parece ser a única solução dos problemas para as crianças e os adolescentes, tornando-se, então, um sério risco de tentativa de suicídio ou suicídio (OMS, 2000). A intensidade desses pensamentos, sua profundidade, bem como o contexto em que surgem e a impossibilidade de desligar-se deles é que são fatores que distinguem um jovem saudável de um que se encontra à margem de uma crise suicida (Borges, Werlang, \& Copatti, 2008).

O desejo de morrer é considerado "o portal" do comportamento autodestrutivo, que representa a inconformidade e a insatisfação do indivíduo com seu modo de vida no momento atual, e se manifesta em frases como: a vida não vale a pena ser vivida; o que deveria fazer é morrer; entre outras (Correa, \& Barrero, 2006b).

A ideação suicida pode ser considerada um fator de risco para o suicídio efetivo, junto com a depressão e a desesperança (Borges, \& Werlang, 2006a) não podendo, portanto, ser menosprezada (Werlang, \& cols., 2005). Atitudes de arrogância e enfrentamento, que procuram demonstrar muita força interior, na realidade, pode ser um pedido de ajuda, de limites, de carinho, de expressão de dúvidas e angústias (Borges, \& cols., 2008).

É preciso ter conhecimento atualizado e sistematizado da frequência de ideação suicida e das tentativas de suicídio, bem como do número de suicídios completos (Palma, Calil, \& Mercadante, 2011), com vistas a elaborar programas de prevenção.

A ideação suicida prediz o ato, fazendo-se necessário não só a detecção precoce desses pensamentos, mas, também, um maior entendimento sobre as causas do seu surgimento e as características peculiares desse período (Araújo, \& cols., 2010).

\section{Dimensão do suicídio no Brasil}

O suicídio está, no mundo inteiro, entre as cinco maiores causas de morte na faixa etária entre 15 e 19 anos sendo que, em vários países, ele fica como primeira ou segunda causa de morte entre meninos e meninas nessa mesma faixa etária.

A Organização Mundial da Saúde, baseada nas tendências atuais estima que, até 2020, aproximadamente 1,53 milhões de pessoas cometerão suicídio, e dez a vinte vezes mais pessoas tentarão suicídio em todo o mundo, representando a média de uma morte a cada 20 segundos e uma tentativa de suicídio a cada 1-2 segundos (Bertolote, \& Fleischmann, 2002).

Segundo dados da OMS (2012), a taxa mundial de suicídio é estimada em torno de 16 por 100 mil habitantes, tendo a taxa de mortalidade por suicídio aumentado $60 \%$ nos últimos 45 anos. Calcula-se que as tentativas de suicídio sejam vinte vezes mais frequentes que o ato consumado.

No Brasil, a taxa geral de mortalidade por suicídio, em 2012, foi de 5,3/100 mil habitantes. O total de suicídios no país, entre os anos 2002 e 2012, passou de 7.726 para 10.321 , representando um aumento de $33,6 \%$, superando o crescimento da população do país no mesmo período, que foi de $11,1 \%$, os homicídios que aumentaram $2,1 \%$ e os óbitos por acidentes de transporte que cresceram $26,5 \%$, (Waiselfisz, 2014).

Segundo o mesmo autor, na população jovem (15 a 29 anos), na qual está incluída a faixa etária final da adolescência (15 a 18 anos), o aumento foi de 15,3\%, passando de 2.515 para 2.900 suicídios entre 2002 e 2012 . A taxa de suicídio, nessa população, passou, no mesmo período, de $5,1 / 100$ mil para $5,6 / 100$ mil jovens, ocupando o país a $60^{\mathrm{a}}$ posição na classificação mundial.

As regiões que apresentaram taxas de suicídio superiores à média nacional de 5,6/100 mil jovens (Unidades Federativas e Capitais) estão listadas na Tabela 1.

A taxa de mortalidade por suicídio na população jovem brasileira, em 2012, foi relativamente baixa $(5,6 / 100$ mil jovens) quando comparada com as taxas de outros países, porém alguns municípios apresentaram índices elevados para o contexto nacional superando, inclusive, as taxas apresentadas pela Rússia $(26,6)$, Cazaquistão $(25,6)$, Bielorrúsia $(25,2)$; Suriname $(21,9)$; e Guiana $(21,5)$, países que encabeçam a lista no nível internacional (Waiselfisz, 2014). Dentre os municípios que ultrapassaram a barreira dos 30 suicídios por 100 mil jovens citam-se: Moju-PA $(41,2)$, CruzAlta-RS $(39,7)$ e Tabatinga-AM $(37,3)$, para uma população jovem de $21.859,15.096$ e 16.077 , respectivamente.

Destaca-se que o município de Tabatinga-AM, que figura como o terceiro lugar na lista de mortalidade suicida entre a população jovem, é um local de assentamento de comunidade indígena, o que eleva a taxa de mortalidade em função do alto índice de suicídio em algumas tribos brasileiras (Waiselfisz, 2014).

Segundo Castro e cols.(2011), a proporção de óbitos por suicídios em 2006 foi de $79 \%$ para o sexo masculino 
Tabela 1 - Taxas de suicídio (em 100 mil) na população jovem (15-29 anos) segundo as regiões do Brasil, 2012.

\begin{tabular}{|c|c|c|c|c|}
\hline \multirow[t]{2}{*}{ REGIÃO } & \multicolumn{4}{|c|}{ TAXAS DE SUICÍDIO } \\
\hline & \multicolumn{2}{|c|}{ UNIDADES FEDERATIVAS } & \multicolumn{2}{|c|}{ CAPITAIS } \\
\hline \multirow[t]{5}{*}{ Norte } & Roraima & 12,9 & Boa Vista & 11,8 \\
\hline & Acre & 10,4 & Rio Branco & 9,5 \\
\hline & Amazonas & 9,5 & Manaus & 7,9 \\
\hline & Tocantins & 9,1 & Macapá & 6,2 \\
\hline & & & Porto Velho & 5,8 \\
\hline \multirow[t]{4}{*}{ Nordeste } & Piauí & 7,9 & Teresina & 10,4 \\
\hline & Ceará & 6,3 & Aracajú & 8,8 \\
\hline & & & Fortaleza & 6,8 \\
\hline & & & João Pessoa & 5,8 \\
\hline \multirow[t]{2}{*}{ Sudeste } & Espírito Santo & 6,5 & Belo Horizonte & 6,0 \\
\hline & Minas Gerais & 6,4 & & \\
\hline \multirow[t]{3}{*}{ Sul } & Rio Grande do Sul & 8,0 & Florianópolis & 7,4 \\
\hline & Paraná & 6,7 & Porto Alegre & 7,4 \\
\hline & Santa Catarina & 6,5 & Curitiba & 6,0 \\
\hline \multirow[t]{3}{*}{ Centro Oeste } & Mato Grosso do Sul & 12,1 & Campo Grande & 9,0 \\
\hline & Mato Grosso & 7,1 & Goiânia & 6,0 \\
\hline & Goiás & 7,1 & & \\
\hline
\end{tabular}

Fonte: Waiselfisz (2014).

(início na faixa dos 15 a 19 anos e pico aos 20 a 29 anos) e de $21 \%$ para o sexo feminino (início na ascendência entre 10 e 14 anos de idade). Esta proporção se mantém ao longo do tempo, reafirmando ser, a mortalidade por suicídio, notadamente masculina.

\section{Prevalência de ideação suicida em adolescentes}

As 33 referências selecionadas, cujas pesquisas incluíram estudantes adolescentes no Brasil e no exterior e tinham por objetivo identificar a prevalência de ideação/ risco suicida em adolescentes da população não clínica bem como os fatores mais significativamente associados estão listadas na Tabela 2.

Observa-se na Tabela 2 que, entre os anos de 2002 a 2013, doze estudos com adolescentes escolares foram desenvolvidos no Brasil, cinco no Chile, três na Colômbia, dois no México, um estudo nos demais países.Considerando que no Brasil a região Sul apresenta alta concentração de suicídio, oito estudos foram realizados naquela região, sendo que em cinco deles a pesquisa foi realizada em escolas (Werlang, \& cols., 2005; Borges, \& Werlang, 2006a; Borges, \& Werlang, 2006b; Borges, \& cols., 2008; Baggio, Palazzo, \& Aerts, 2009), e em três, foi realizada fora do ambiente escolar (Souza, \& cols., 2010a;Souza, \& cols., 2010b; Ores, \& cols., 2012).

Verifica-se que as prevalências encontradas em cinco estudos realizados no Brasil, variaram entre $31,9 \%$ e
36,0\% (Werlang, \& cols., 2005; Borges, \& Werlang, 2006a; Borges, \& Werlang, 2006b; Jatobá,\& Bastos, 2007; Borges, \& cols.,2008), resultados similares ao encontrado por Salvo Garrido e Castro Salvo (2013) em estudo realizado no Chile, e abaixo dos resultados encontrados no Equador, Chile e México (Salvo Garrido, \& Melipillán Araneda, 2008; Villarreal, 2009; Ventura-Juncá Domínguez, \& cols.,2010; Perez-Amezcua, \& cols., 2010).

Um estudo realizado com adolescentes estudantes do ensino fundamental e médio da cidade João Pessoa-PB apresentou prevalência de 22,2\% para ideação suicida (Araújo, \& cols., 2010), resultado próximo aos encontrados em estudos realizados na Colômbia, Chile, Peru e Uganda (Carvajal, \& Caro, 2009; Barroilhet, \& cols., 2012; Cano, \& cols., 2009; Rudatsikira, Muula, Siziya, \& Twa-Twa, 2007).

A prevalência de planejamento suicida encontrada por Botega e cols., (2009), de 5,3\%, foi a mais baixa de todos os estudos realizados no Brasil, entretanto, o resultado apresentado corresponde à ideação suicida nos últimos 12 meses. Tal achado pode ser em decorrência de que, no que tange à ideação suicida ao longo da vida, as prevalências encontradas na literatura científica são mais elevadas (Souza, \& cols., 2010a).

Apesar de a literatura afirmar que a prevalência de ideação suicida ao longo da vida é mais elevada, observando-se a Tabela 2 , verifica-se que as investigações realizadas com adolescentes não revelaram diferenças significativas entre os resultados de ideação suicida, tendo sido encontradas prevalências que variavam de: $12,1 \%$ a $62 \%$ ao 
Tabela 2. Prevalência e fatores associados à ideação/risco suicida na adolescência, 2002-2013.

\begin{tabular}{|c|c|c|c|c|}
\hline ESTUDO & PAÍS & SUJEITOS DA PESQUISA & PREVALÊNCIA & $\begin{array}{c}\text { FATORES } \\
\text { ALTAMENTE } \\
\text { SIGNIFICATIVOS }\end{array}$ \\
\hline Freitas e Botega (2002) & Brasil & Adolescentes de 14 a 18 anos & $16,7 \%$ & $\begin{array}{l}\text { Depressão; preocupação; pouco } \\
\text { apoio social. }\end{array}$ \\
\hline Werlang e cols. (2005) & Brasil & Adolescentes de 15 a 19 anos & $* 35,7 \%$ & $\begin{array}{l}\text { Depressão; conhecer pessoa que } \\
\text { tenha tentado suicídio. }\end{array}$ \\
\hline $\begin{array}{l}\text { Borges e Werlang } \\
(2006 b)\end{array}$ & Brasil & Adolescentes de 13 a 19 anos & $34,7 \%$ & Depressão; sexo feminino. \\
\hline Jatobá e Bastos (2007) & Brasil & Adolescentes de 14 a 16 anos & $34,3 \%$ & Sintomas depressivos; ansiedade. \\
\hline $\begin{array}{l}\text { Rudatsikira, Muula e } \\
\text { Siziya } \\
(2007)\end{array}$ & Guiana & Adolescentes $<14 \mathrm{a} \geq 16$ anos & ** $18,4 \%$ & $\begin{array}{l}\text { Ter sido maltratado; consumo de } \\
\text { álcool; depressão; tabagismo. }\end{array}$ \\
\hline Borges e cols. (2008) & Brasil & Adolescentes de 13 a 17 anos & 31,9 & Depressão; sexo feminino. \\
\hline $\begin{array}{l}\text { Granero, Poni e Poni } \\
\text { (2008) }\end{array}$ & Venezuela & Adolescentes de 12 a 15 anos & $* * 13,5 \%$ & $\begin{array}{l}\text { Preocupação; relação sexual; } \\
\text { solidão; poucos amigos próximos; } \\
\text { consumo de álcool. }\end{array}$ \\
\hline $\begin{array}{l}\text { Salvo Garrido e } \\
\text { Melipillán Araneda } \\
(2008)\end{array}$ & Chile & Adolescentes de 14 a 19 anos & $* * 43,3 \%$ & $\begin{array}{l}\text { Idade, impulsividade; sexo feminino; } \\
\text { baixa } \\
\text { autoestima; problemas de coesão } \\
\text { familiar. }\end{array}$ \\
\hline \multirow[t]{2}{*}{ Carvajal e Caro (2009) } & Colômbia & Adolescentes de 14 a 17 anos & $\# 20,0 \%$ & $\begin{array}{l}\text { Antecedente de tentativa de } \\
\text { suicídio; depressão; desesperança; } \\
\text { solidão; }\end{array}$ \\
\hline & & & & $\begin{array}{l}\text { baixa autoestima; consumo de } \\
\text { álcool e substâncias psicoativas. }\end{array}$ \\
\hline Botega e cols. (2009) & Brasil & $\begin{array}{l}\text { Indivíduos com idade } \geq 14 \\
\text { anos }\end{array}$ & * $17,1 \%$ **5,3\% & Depressão; ansiedade; sexo feminino; solteiro. \\
\hline Lerech e cols. (2009) & Colômbia & Estudantes de 8 a 20 anos & $12,8 \%$ & $\begin{array}{l}\text { Depressão; ansiedade; disfunções familiares; } \\
\text { sexo feminino. }\end{array}$ \\
\hline $\begin{array}{l}\text { Villarreal } \\
(2009)\end{array}$ & Equador & Adolescentes de 12 a 19 anos & $39,3 \%$ & $\begin{array}{l}\text { Disfunção familiar, migração } \\
\text { materna. }\end{array}$ \\
\hline Araújo e cols. (2010) & Brasil & Adolescentes de 14 a 18 anos & $22,2 \%$ & Solidão; desesperança; sofrimento psíquico. \\
\hline $\begin{array}{l}\text { Perez-Amezcua e cols. } \\
\text { (2010) }\end{array}$ & México & Adolescentes de 14 a 19 anos & $47,0 \%$ & $\begin{array}{l}\text { Pouca comunicação com pais; sintomas } \\
\text { depressivos; consumo de álcool e drogas; } \\
\text { ansiedade; sexo feminino. }\end{array}$ \\
\hline Souza e cols. (2010a) & & Adolescentes de 15 a 18 anos & $* * 7,7 \%$ & $\begin{array}{l}\text { Agressividade; sexo feminino; consumo de álcool } \\
\text { e drogas. }\end{array}$ \\
\hline Souza e cols. (2010b) & Brasil & Adolescentes de 11 a 15 anos & $14,1 \%$ & $\begin{array}{l}\text { Depressão; sexo feminino; consumo de álcool e } \\
\text { drogas. }\end{array}$ \\
\hline
\end{tabular}


(continuação)

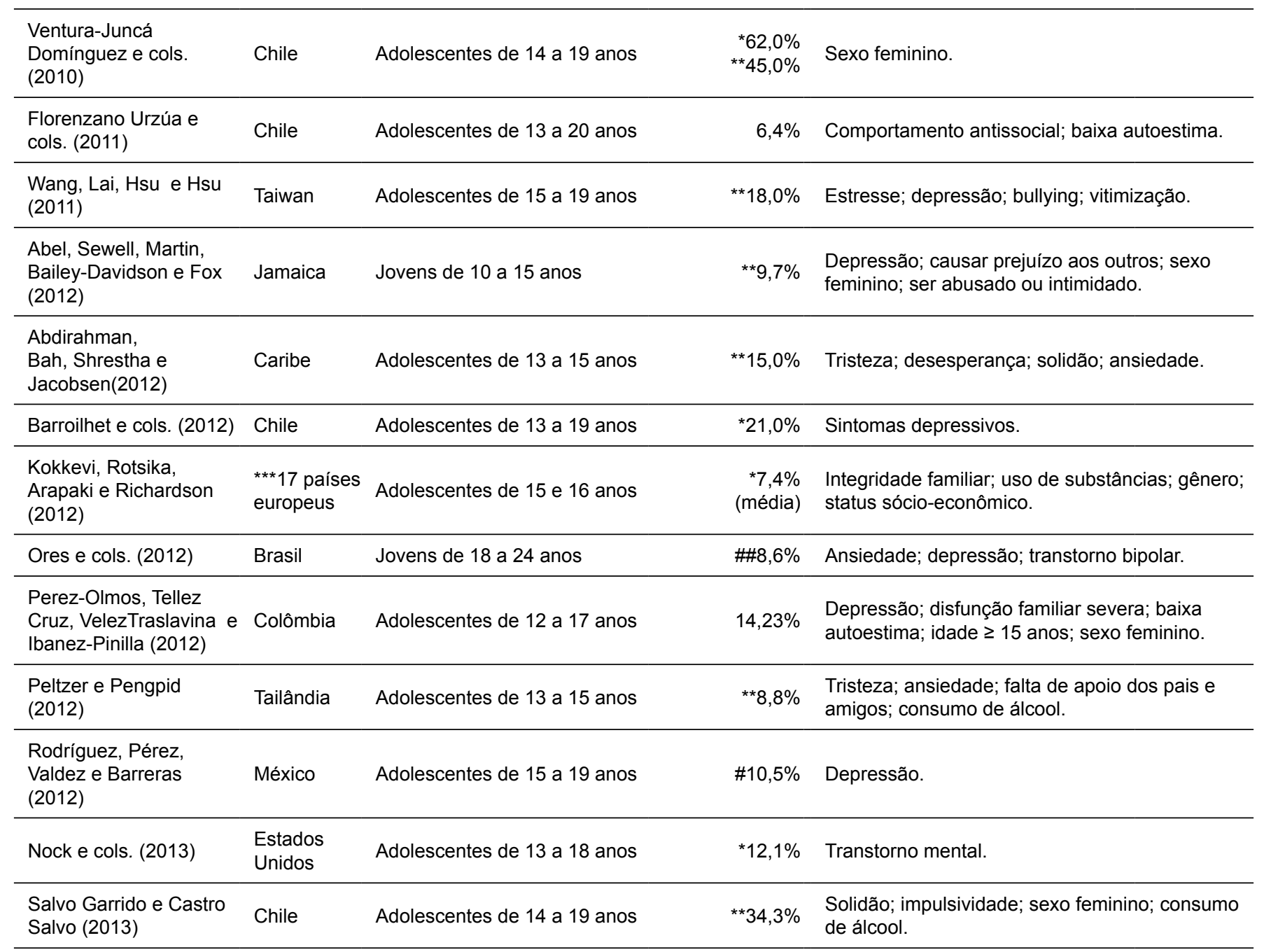

Nota:Ideação: * longo da vida; ** últimos 12 meses; \# última semana; \#\# últimos 3 meses.

*** Países participantes do Projeto Europeu de Inquérito Escolar sobre Álcool e outras Drogas: Hungria, Chipre, Látvia, Ilha de Man, Grécia, Eslovênia, Áustria, Ilhas Faroé, Islândia, Romênia, Bulgária, Reino Unido, Croácia, Ucrânia, República Eslovaca, Bélgica (Flandres) e Armênia.

longo da vida; $5,3 \%$ a $45 \%$ nos últimos 12 meses; e, 10,5\% a $20 \%$ na última semana.

Além da diferença temporal para a ideação suicida, outra explicação para a variação entre os dados de prevalência encontrados pode ser as diferenças metodológicas na aferição da ideação suicida, na seleção da amostra, além dos fatores socioculturais (Souza, \& cols., 2010a). Apesar do amplo uso das taxas, quando usadas isoladamente, as informações transmitidas por elas podem ser enganosas, particularmente quando são comparados dados de países ou regiões com importantes diferenças na estrutura demográfica (Bertolote, \& Fleischmann, 2002).

Os fatores associados à ideação suicida na adolescência, encontrados nos estudos analisados, são multifacetados e incluem transtornos mentais, características pessoais e familiares, problemas comportamentais do próprio adolescente e dos amigos. Dentre os fatores que mais sobressaíram destaca-se: depressão, desesperança, solidão, tristeza, preocupação, ansiedade, baixa autoestima, agressão por parte de pais e amigos, pouca comunicação com os pais, ser abusado fisicamente na escola, uso de substâncias, pessoa conhecida com tentativa de suicídio, e, pertencer ao sexo feminino.

A depressão acomete, ao longo da vida, entre $10 \%$ a $15 \%$ das mulheres e, entre $5 \%$ a $12 \%$ dos homens, sendo que, entre os gravemente deprimidos, $15 \%$ cometem suicídio (Brasil, 2006b). Os dados disponíveis demonstram que a prevenção e o tratamento adequados da depressão e do abuso de álcool e substâncias psicoativas reduzem as taxas de suicídio (OMS, 2012).

A prevenção do suicídio dá-se por meio do reforço dos fatores ditos protetores e pela diminuição dos fatores de risco, tanto no nível individual como no coletivo (Araújo, \& cols., 2010). Para a OMS (2000) são fatores protetores: bom 
relacionamento com os familiares e apoio familiar (fatores familiares); boas habilidades/relações sociais, confiança em si mesmo, buscar e estar aberto a conselhos (personalidade e estilo cognitivo); integração social, bom relacionamento com colegas de escola, com professores e outros adultos, ajuda de pessoas relevantes (fatores culturais, sociais e demográficos).

\section{Considerações Finais}

Analisando os dados obtidos nota-se que, nas últimas décadas, houve um crescimento na ocorrência de suicídio na fase da adolescência. Considerado um sério problema de saúde pública, o suicídio na adolescência traz consequências negativas não só para a família da vítima, mas também para o meio social no qual o adolescente está inserido.

Os dados coletados nas diversas referências analisadas revelam que a prevalência de ideação suicida não apresenta diferença significativa entre os países, demonstrando que os adolescentes do mundo todo estão necessitando de ajuda para evitar que suas vidas sejam perdidas. Os estudos indicam que as prováveis motivações para a ideação suicida (histórico de suicídio na família, transtornos mentais, exposição à violência, abuso de álcool e drogas, conflitos familiares) tendem a ser constante nos adolescentes de diferentes culturas.

A presença de sintomas depressivos destacou-se como um importante fator de risco para a ideação suicida nos estudos analisados, e, considerando que os dados se referem a uma população não-clínica de adolescentes, torna-se ainda mais preocupante os achados dos pesquisadores. Os estudos analisados corroboram as informações, mencionadas na literatura especializada, que salientam que os adolescentes do sexo feminino apresentam taxas mais elevadas de ideação suicida que os do sexo masculino. Este fato pode ser explicado em razão de a mulher ser mais vulnerável a transtornos como depressão e ansiedade, fatores estes que afetam fortemente a ideação suicida.

Considerando que os estudos realizados no Brasil indicam uma taxa elevada de ideação suicida na população adolescente, resultado de uma gama de fatores de risco a que tal população está exposta e, que diferenças regionais, culturais, socioeconômicas, entre outras, influenciam o índice de prevalência de ideação suicida, faz-se necessário o desenvolvimento de estudos semelhantes em diferentes cidades e regiões do país, de forma a identificar outras variáveis associadas ao comportamento suicida, vez que a perda prematura de adolescentes por suicídio pode e deve ser evitada.

Como as pesquisas foram realizadas com estudantes, sendo a maioria delas no ambiente escolar, é importante que os profissionais da educação, em conjunto com outros profissionais como médicos, psicólogos, enfermeiros e assistentes sociais, participem de programas de capacitação continuada, além do planejamento de ensino anual, que vise à otimização da comunicação entre os atores escolares em assuntos relacionados à vida, morte, sofrimento, depressão e condutas autodestrutivas nessa população.

Países como Estados Unidos e Austrália já desenvolvem esforços na tentativa de criar centros de prevenção e programas que possam dar conta da prevenção do suicídio na adolescência. Visando reduzir as taxas de suicídios e tentativas, bem como os danos associados a comportamentos suicidas, elaborou-se a Estratégia Nacional de Prevenção do Suicídio (ENPS), instituída pela Portaria do Ministério da Saúde $n^{\circ}$ 1.876, de 14 de agosto de 2006 (Brasil, 2006a), envolvendo uma série de ações de prevenção e promoção de saúde, que vão desde a melhora das condições de vida e dos vínculos familiares e comunitários até a oferta de uma rede de serviços de saúde e de saúde mental que ofereçam tratamento e reabilitação psicossocial.

Podemos dizer que o Brasil avançou, porém necessita de maiores investimentos na criação de programas e estratégias específicas voltadas à prevenção do comportamento suicida na população jovem e adolescente.

\section{Referências}

Abdirahman, H. A., Bah, T. T., Shrestha, H. L., \& Jacobsen, K. H. (2012). Bullying, mental health, and parental involvement among adolescents in the Caribbean.West Indian Medical Journal, 61(5), 504-508.

Abel, W. D., Sewell, C., Martin, J. S., Bailey-Davidson, Y., \& Fox, K. (2012). Suicide ideation in Jamaican youth: sociodemographic prevalence, protective and risk factors. West Indian Medical Journal, 61(5), 521-525.

Araújo, L. C., Vieira, K. F. L., \& Coutinho, M. P. L. (2010). Ideação suicida na adolescência: um enfoque psicossociológico no contexto do ensino médio. Psicologia - Universidade São Francisco, 15(1), 47-57.

Baggio, L., Palazzo, L. S., \& Aerts, D. R. G. C. (2009). Planejamento suicida entre adolescentes escolares: prevalência e fatores associados. Cadernos de Saúde Pública, 25(1), 142-150.

Barroilhet, S., Fritsch, R., Guajardo, V., Martínez, V., Vöhringer, P., Araya, R.,\& Rojas, G.(2012). Ideas autolíticas, violencia autoinfligida, y síntomas depresivos en escolares chilenos. Revista Médica de Chile, 140(7), 873-881.

Bertolote, J. M., \& Fleischmann, A. (2002).A global perspective in the epidemiology of suicide.Suicidologi., 7(2), 6-8.

Borges, V. R., \& Werlang, B. S. G. (2006a). Estudo de ideação suicida em adolescentes de 15 a 19 anos. Estudos de Psicologia, 11(3), 345-351.

Borges, V. R., \& Werlang, B. S. G. (2006b). Estudo de ideação suicida em adolescentes de 13 a 19 anos. Psicologia, Saúde e Doenças, 7(2), 195-209. 
Borges, V. R., Werlang, B. S. G., \& Copatti, M. (2008).Ideação suicida em adolescentes de 13 a 17 anos. Barbarói, 28.

Botega, N. J., Marín-León, L., Oliveira, H. B., Barros, M. B. A., Silva, V. F., \& Dalgalarrondo, P. (2009). Prevalências de ideação, plano e tentativa de suicídio: um inquérito de base populacional em Campinas, São Paulo, Brasil. Cadernos de Saúde Pública, 25(12), 2632-2638.

Brasil. Ministério da Saúde. (2006a). Portaria $n^{\circ}$ 1.876, de 14 de agosto de 2006. Diário Oficial da União, Brasília-DF, 15 agosto 2006. Seção 1, p. 65.

Brasil. Ministério da Saúde. (2006b). Prevenção do suicídio: manual dirigido a profissionais das equipes de saúde mental. Organização Pan-Americana da Saúde. Universidade Estadual de Campinas. Recuperado: 11 nov. 2012. Disponível:http://portal.saude.gov.br/ portal/arquivos/pdf/manual_prevencao_suicidio_saude_mental. pdfem.

Cano, P., Gutiérrez, C., \& Nizama, M. (2009). Tendencia a la violencia e ideación suicida en adolescentes escolares en una ciudad de la Amazonía Peruana.Revista Peruana de Medicina Experimental y Salud Publica, 26(2),175-181.

Carvajal, G., \& Caro, C.V. (2011). Ideación suicida en la adolescencia: una explicación desde tres de sus variables asociadas en Bogotá, 2009. Colombia Médica, 42(2,Supl. 1), 45-56.

Castro, M. L., Cunha, S. S., \& Souza, D. P. O. (2011). Comportamento de violência e fatores associados entre estudantes de Barra do Garças, MT. Revista Saúde Pública, 45(6), 1054-1061.

Correa, H., \& Barrero, S. P. (2006a). O suicídio ao longo dos tempos. Em H. Correa, \& S. P. Barrero (Orgs.), Suicídio uma morte evitável (pp. 3-10). São Paulo, Atheneu.

Correa, H., \& Barrero, S. P. (2006b). O suicídio: definições e classificações. Em H. Correa, \& S. P. Barrero (Orgs.), Suicídio uma morte evitável (pp. 29-36). São Paulo, Atheneu.

Florenzano Urzúa, R., Valdés Correa, M., Cáceres Contreras, E., Santander Rigollet, S., Aspillaga Herrera, C., \& Musalem Sarquis, C. (2011). Relación entre ideación suicida y estilos parentales en un grupo de adolescentes chilenos. Revista Médica de Chile, 139(12), 1529-1533.

Freitas, G. V. S., \& Botega, N. J. (2002). Gravidez na adolescência: prevalência de depressão, ansiedade e ideação suicida. Revista da Associação Médica Brasileira, 48(3), 245-249.

Granero, R.,Poni, E., \& Poni, C. (2008). Suicidal ideation among students of the 7th, 8th, and 9th grades in the State of Lara, Venezuela: the Global School Health Survey. Puerto Rico Health Sciences Journal, 27(4), 337-342.
Jatobá, J. A. V. N., \& Bastos, O. (2007). Depressão e ansiedade em adolescentes de escolas públicas e privadas. Jornal Brasileiro de Psiquiatria, 56(3).

Kokkevi, A., Rotsika, V., Arapaki, A., \& Richardson, C. (2012). Adolescents' self-reported suicide attempts, self-harm thoughts and their correlates across 17 European countries. Journal of Child Psychology and Psychiatry,53(4), 381-389.

Lerech, M. M. F., Arias, A. F. G., Castrillon, J. J. C., Arias, C. F. H., Campoalegre, P. A. O., Cala, M. L. P., Garzón, D. M. P., \& Garcia, L. M. Z. (2009). Riesgo suicida y factores relacionados en Estudiantes de $6^{\circ}$ a $11^{\circ}$ grado en colegios de la ciudad de Manizales (Colombia): 2007-2008. Archivos de Medicina, 9(2).

Lovisi, G. M., Santos, S. A., Legay, L., Abelha, L., \& Valencia, E. (2009). Análise epidemiológica do suicídio no Brasil entre 1980 e 2006. Revista Brasileira de Psiquiatria, 31(Supl. II), 86-93.

Nock, M. K., Green, J. G., Hwang, I., McLaughlin, K. A., Sampson, N. A., Zaslavsky, A. M., \& Kessler, R. C. (2013).Prevalence, Correlates, and Treatment of Lifetime Suicidal Behavior Among Adolescents. Results From the National Comorbidity Survey Replication Adolescent Supplement. JAMA Psychiatry, 70(3), 300-310.

Ores, L. C., Quevedo, L. A., Jansen, K., Carvalho, A. B., Cardoso, T. A., Souza, L. D. M., Pinheiro, R. T. , \& Silva, R. A. (2012). Risco de suicídio e comportamentos de risco à saúde em jovens de 18 a 24 anos: um estudo descritivo. Cadernos de Saúde Pública, $28(2), 305-312$.

Organização Mundial da Saúde [OMS] (2000). Departamento de Saúde Mental. Transtornos Mentais e Comportamentais. Prevenção do suicídio: manual para professores e educadores. Recuperado: 27 nov.2012. Disponível: http://apps.who.int/iris/ bitstream/10665/66801/5/WHO_MNH_MBD_00.3_por.pdf.

Organização Mundial da Saúde [OMS] (2012). Salud mental. Prevención del suicidio (SUPRE). Recuperado: 10 jul. 2013. Disponível: http://www.who.int/mentl_health/prevention/suicide/ suicideprevent/es/index.html.

Palma, S. M. M., Calil, H. M., \& Mercadante, M. T. (2011). Suicídio em adolescentes no Brasil: problema de saúde pública? Carta aos editores. Revista Brasileira de Psiquiatria, 33(1).

Peltzer, K., \& Pengpid, S. (2012). Suicidal Ideation and Associated Factors among School-Going Adolescents in Thailand. International Journal of Environmental Research and PublicHealth, 9(2), 462473.

Pérez-Amezcua, B., Rivera-Rivera, L., Atienzo, E. E., De Castro, F., Leyva-López, A., \& Chávez-Ayala, R. (2010). Prevalencia y factores asociados a la ideación e intento suicida en adolescentes de educación media superior de la República mexicana. Salud Pública de México, 52(4), 324-333. 
Perez-Olmos, I., Tellez Cruz, D. L., Velez Traslavina, A. L.,\& IbanezPinilla, M. (2012). Caracterización de factores asociados con comportamiento suicida en adolescentes estudiantes de octavo grado, en tres colegios bogotanos. Revista Colombiana de Psiquiatría, 41(1), 26-47.

Rodríguez, M. J. C., Pérez, R. R., Valdez, E. A., \& Barreras, A. L. G. (2012). Depresión y comportamiento suicida en estudiantes de educación media superior en Sonora. Salud Mental, 35(1), 45-50.

Rudatsikira, E., Muula, A. S.,\& Siziya, S. (2007). Prevalence and associated factors of suicidal ideation among school-going adolescents in Guyana: results from a cross sectional study. Clinical Practice and Epidemiology in Mental Health, 3.

Rudatsikira, E., Muula, A. S.,Siziya, S., \& Twa-Twa, J. (2007). Suicidal ideation and associated factors among school-going adolescents in rural Uganda. BioMed Central Psychiatry, 7.

Salvo Garrido, L., \& Melipillán Araneda, R. (2008). Predictores de suicidalidad en adolescentes. Revista Chilena de Neuropsiquiatría, 46(2), 115-123.

Salvo Garrido, L., \& Castro Salvo, A. (2013).Soledad, impulsividad, consumo de alcohol y su relación con suicidalidad en adolescentes. Revista Médica de Chile, 141(4), 428-434.

Souza, L. D. M., Ores, L., Oliveira, G. T., Cruzeiro, A. L. S., Silva, R. A., Pinheiro, R. T., \& Horta, B.L. (2010a). Ideação suicida na adolescência: prevalência e fatores associados. Jornal Brasileiro de Psiquiatria, 59(4), 286-292.
Souza, L. D. M., Silva, R. A., Jansen, K., Kuhn, R. P., Horta, B. L., \& Pinheiro, R. T. (2010b). Suicidal ideation in adolescents aged 11 to 15 years: prevalence and associated factors. Revista Brasileira de Psiquiatria, 32(1), 37-41.

Ventura-Juncá Domínguez, R., Carvajal, C., Undurraga, S., Vicuña, P., Egaña, J., \& Garib, M. J. (2010). Prevalencia de ideación e intento suicida en adolescentes de la Región Metropolitana de Santiago de Chile. Revista Médica de Chile, 138(3), 309-315.

Villarreal, D. L. (2009). Prevalencia de ideación suicida y factores asociados en adolescentes de los colegios urbanos de la ciudad de Cuenca. Tese de Mestrado em Saúde Pública, Facultad de Ciencias Médicas, Universidad de Cuenca, Cuenca-Ecuador.

Waiselfisz, J. J. (2014). Mapa da violência 2014: os jovens do Brasil. Brasilia.

Wang, R. H., Lai, H. J., Hsu, H. Y., \& Hsu, M. T. (2011).Risk and protective factors for suicidal ideation among Taiwanese adolescents. Nursing Research, http://journals.Iww.com/ nursingresearchonline/toc/2011/11000

Werlang, B. S. G., Borges, V. R. \& Fensterseifer, L. (2005). Fatores de risco ou proteção para a presença de ideação suicida na adolescência. Revista Interamericana de Psicologia, 39(2), 259266.

\section{Sobre os autores}

Lenice Carrilho de Oliveira Moreira (lenice.moreira@ufms.br)

Administradora na Universidade Federal de Mato Grosso do Sul. Mestre em Tecnologias Ambientais.

Paulo Roberto Haidamus de Oliveira Bastos (phaidamus43@gmail.com)

Professor Associado 4 da Universidade Federal de Mato Grosso do Sul. Doutor em Educação. 
